\title{
Corrigendum: Long-term persistence and development of induced pancreatic beta cells generated by lineage conversion of acinar cells
}

Weida Li, Claudia Cavelti-Weder, Yinying Zhang, Kendell Clement, Scott Donovan, Gabriel Gonzalez, Jiang Zhu, Marianne Stemann, Ke Xu, Tatsu Hashimoto, Takatsugu Yamada, Mio Nakanishi, Yuemei Zhang, Samuel Zeng, David Gifford, Alexander Meissner, Gordon Weir \& Qiao Zhou

Nat. Biotechnol. 32, 1223-1230 (2014); published online 17 November 2014; corrected after print 14 May 2015

In the version of this article initially published, Yingying Zhang's name was spelled Yinying Zhang. The error has been corrected in the HTML and PDF versions of the article.

\section{Corrigendum: The ethics of publishing human germline research}

Arun Sharma \& Christopher Thomas Scott

Nat. Biotechnol. 33, 590-592 (2015); published online 9 June 2015; corrected after print 9 June 2015

In the version of this article initially published, a name in the acknowledgments was spelled as Vittoria, rather than Vittorio, Sebastiano. The error has been corrected in the HTML and PDF versions of the article.

\section{Corrigendum: Suppression of vascular permeability and inflammation by targeting of the transcription factor c-Jun}

\begin{abstract}
Roger G Fahmy, Alla Waldman, Guishui Zhang, Ainslie Mitchell, Nicodemus Tedla, Hong Cai, Carolyn R Geczy, Colin N Chesterman, Michael Perry \& Levon M Khachigian

Nat. Biotechnol. 24, 856-863 (2006); published online 2 July 2006; corrected after print 10 June 2015

In the version of this article initially published, the first three bars in the histogram in Figure 1a should have read "No vehicle," "No Dz" and "Dz13" instead of "No Dz," "Dz13" and "Dz13scr." The legend of Figure 1a should have included the sentences: "No vehicle' represents the normoxia control without vehicle (transfection agent) or DNAzyme or siRNA. All other groups contain vehicle." The H\&E-stained images in Figure 1a should have read "Dz13 in hyperoxia-normoxia" and "Dz13scr in hyperoxia-normoxia" instead of "Normoxia" and "Hyperoxia-normoxia." None of the conclusions is affected by the errors. The errors have been corrected in the HTML and PDF versions of the article.
\end{abstract}

\section{Erratum: A mass-tolerant database search identifies a large proportion of unassigned spectra in shotgun proteomics as modified peptides}

Joel M Chick, Deepak Kolippakkam, David P Nusinow, Bo Zhai, Ramin Rad, Edward L Huttlin \& Steven P Gygi

Nat. Biotechnol. doi:10.1038/nbt.3267; corrected online 18 June 2015

In the version of this article initially published online, in Figure $3 \mathrm{c}$, the label "unidentified b-type ions" should have been deleted, and in Figure 4, the symbols over the peptide sequences were misplaced. The errors have been corrected for the print, PDF and HTML versions of this article.

\section{Erratum: Public biotech 2013-the numbers}

Stacy Lawrence \& Riku Lahteenmaki

Nat. Biotechnol. 32, 626-632 (2014); published online 8 July 2014; corrected after print 3 September 2014 and 25 June 2015

In the version of the article initially published, some companies that went public in 2013 were missing from the table of 2013 IPOs (Table 4). Thirteen companies have been added to the table, including Celyad (formerly Cardio3 Biosciences), Erytech, Immunicum, Innate Immune Therapeutics, Kadimastem, Karyopharm Therapeutics, Kindred, Macrogenics, Oncolys Biopharma, Reprocell, Tetralogic, Veracyte and Xencor; in the figure of global IPOs (Fig. 2), the number of IPOs in 2013 and the average dollar amount raised have been corrected to reflect the additional companies. 